\title{
TENDENCIAS EVOLUTIVAS DEL CONTENIDO DIGITAL EN APLICACIONES MÓVILES
}

\section{Evolutionary trends of digital content in mobile applications}

Juan-Miguel Aguado, Inmaculada J. Martínez y Laura Cañete-Sanz

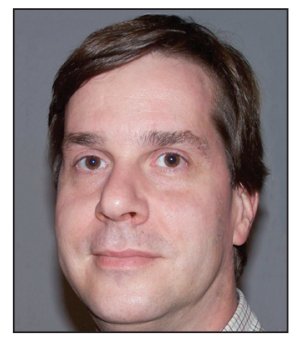

Juan-Miguel Aguado es doctor en ciencias de la información por la Universidad Complutense de Madrid y posgrado en investigación social por la Polish Academy of Sciences (Varsovia). Profesor titular de teoría de la comunicación en la Facultad de Comunicación y Documentación de la Universidad de Murcia, co-dirige el proyecto I+D Comunicación móvil e información personal: impacto en la industria del contenido, el sistema publicitario y el comportamiento de los usuarios financiado por el Plan Nacional de I+D (CSO2013-47394-R) y es director del proyecto MOB AD: El impacto de la tecnología móvil en la comunicación estratégica y publicitaria financiado por la Fundación Séneca (19451/PI/14).

http://orcid.org/0000-0002-8922-3299

jmaguado@um.es

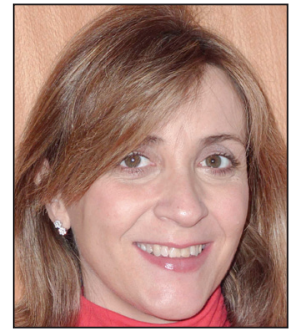

Inmaculada J. Martínez es doctora en ciencias de la información por la Universidad Complutense de Madrid y master en dirección y gestión de empresas por la Know How Business School. Profesora titular de empresa publicitaria en la Facultad de Comunicación y Documentación de la Universidad de Murcia, ha realizado consultoría y formación sobre estrategias de imagen y comunicación en España, Brasil y Portugal. Co-dirige el proyecto Comunicación móvil e información personal: impacto en la industria del contenido, el sistema publicitario y el comportamiento de los usuarios financiado por el Plan Nacional de I+D (CSO2013-47394-R) y participa en el proyecto MOB AD: El impacto de la tecnología móvil en la comunicación estratégica y publicitaria financiado por la Fundación Séneca (19451/PI/14).

http://orcid.org/0000-0003-3807-1325

inmartin@um.es

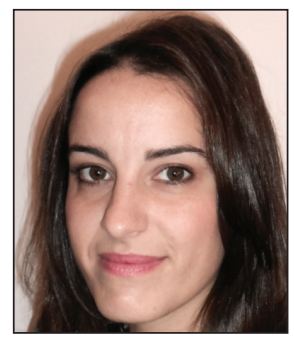

Laura Cañete-Sanz es investigadora y becaria FPI en el proyecto Comunicación móvil e información personal: impacto en la industria del contenido, el sistema publicitario y el comportamiento de los usuarios financiado por el Plan Nacional de I+D (CSO2013-47394-R). Es licenciada en periodismo, en publicidad y RR.PP. y diplomada en biblioteconomía y documentación por la Universidad de Murcia, así como Máster en Estudios Avanzados en Comunicación por la misma universidad. Es miembro del Grupo de Investigación en Comunicación Social, Cultura y Tecnología (E-COM). http://orcid.org/0000-0003-4515-1673

laura.canete@um.es

Universidad de Murcia, Facultad de Comunicación y Documentación Campus de Espinardo, s/n. 30100 Murcia, España

\section{Resumen}

En los últimos años las aplicaciones móviles han constituido un ecosistema propio y un potente motor de innovación. La consolidación de las aplicaciones móviles como interfaz dominante del acceso a contenido tiene consecuencias en el plano de la producción, la distribución y el consumo, fusionando los cuatro ámbitos funcionales característicos del contenido digital: reproducción, creación, gestión y comunicación. En este artículo se fundamenta una tipología funcional de las aplicaciones móviles de contenido y un modelo de análisis evolutivo. A partir de él se analiza la evolución funcional de las 153 versiones desarrolladas durante dos años (marzo de 2013 a marzo de 2015) para una muestra de 12 aplicaciones significativas. Aunque la muestra ofrece una representatividad limitada, los resultados permiten validar el modelo y apuntan a que la capacidad de intervención y la capacidad de comunicación sobre el contenido constituyen elementos importantes en la creación de valor en las aplicaciones móviles de contenido, si bien no de forma homogénea en todos los tipos de aplicaciones establecidos. 


\title{
Palabras clave
}

Contenido móvil; Aplicaciones; Internet móvil; Industrias culturales; Evolución funcional; Actualizaciones.

\begin{abstract}
In recent years, mobile applications have come to constitute an ecosystem of their own, becoming an important driver for innovation. The consolidation of mobile applications as the prevalent interface for content access has consequences for the production, distribution and consumption of content, merging the functional spheres of digital content: performance, creation, management and communication. This paper proposes a functional typology of mobile content applications, along with an evolutionary analysis model. It analyses the functional evolution of 153 versions of 12 relevant applications developed during two years (March 2013 through March 2015). Though statistical representativeness is limited, the sample analysis allowed validation of the model. The results point to intervention capacity and communication capacity over content as important value-added drivers in mobile content applications, although not in homogeneous terms for all the kinds of mobile applications analysed.
\end{abstract}

\section{Keywords}

Mobile content; Applications; Mobile internet; Cultural industries; Functional evolution; Updates.

Aguado, Juan-Miguel; Martínez, Inmaculada J.; Cañete-Sanz, Laura (2015). "Tendencias evolutivas del contenido digital en aplicaciones móviles". El profesional de la información, v. 24, n. 6, pp. 787-795.

http://dx.doi.org/10.3145/epi.2015.nov.10

\section{Introducción}

Sólo dos años después de que Apple lanzara su AppStore y Google pusiera en marcha el Android Market, los editores de la revista Wired publicaban un artículo titulado "La Web ha muerto, larga vida a internet" (Anderson; Wolff, 2010). En él aventuraban un cambio en el uso de internet, hasta entonces centrado en la Web abierta y basado en buscadores y navegadores, que según los autores, evolucionaría rápidamente hacia un modelo de plataformas semi-cerradas basado en aplicaciones que gestionan los datos online y prescinden del navegador.

\section{El uso de internet en dispositivos móvi- les ha evolucionado rápidamente hacia las aplicaciones en detrimento de los na- vegadores}

Cinco años después la Web no ha muerto, pero el uso de aplicaciones ha crecido de manera exponencial. Del total del tiempo dedicado a medios, los dispositivos móviles disputan la posición preeminente de la televisión o el PC, con una media diaria cercana al $40 \%$ del total -por encima de las dos horas y media diarias (Meeker, 2014; Khalaf, 2014)-. El uso de internet en dispositivos móviles ha evolucionado rápidamente hacia las aplicaciones en detrimento de los navegadores: en 2014 sólo un 14\% del uso de internet móvil implicaba navegadores, mientras que el $86 \%$ era a través de aplicaciones (Khalaf, 2014). Del uso mediante aplicaciones, un $75 \%$ supone alguna forma de comunicación y/o acceso a contenido (Khalaf, 2014). La dimensión estratégica en el entorno móvil del contenido y servicios asociados es sólo comparable al impacto que la tecnología y los nuevos actores de la movilidad tienen en las industrias del contenido, inmersas todavía en un doloroso proceso de adaptación al mundo digital (De-Prato; Sanz; Simon, 2014).
La implantación vertiginosa de las aplicaciones en el entorno móvil obedece a:

- razones de economía funcional: aportan valor en la relación tiempo/eficacia, algo especialmente relevante en la pequeña pantalla ubicua (Scolari; Aguado; Feijóo, 2012); - criterios estratégicos de mercado.

Como ha señalado Fransman (2014), mediante el ecosistema de aplicaciones móviles las nuevas plataformas (como Apple/iOS o Google/Android) consiguen dos objetivos:

- con las tiendas de aplicaciones y contenido (App Store y iTunes o Google Play) se garantizan el control de un canal de distribución dominante que les permite articular servicios de valor añadido (plataformas publicitarias, servicios de sincronización y almacenamiento en la nube, pago y facturación, métricas de usuarios, etc.) (De-Prato; Sanz; Simon, 2014);

- con el ecosistema de aplicaciones, las plataformas producen un entorno de innovación emergente (Fransman, 2014) basado en los principios de la evolución -cantidad, diversidad y selección-.

Mediante el estímulo a los desarrolladores (porcentajes del $70 \%$ sobre los ingresos, kits de desarrollo, gestión de la visibilidad, servicios de valor añadido...), son capaces de producir una gran y diversa población de aplicaciones.

Al mismo tiempo las plataformas estimulan la capacidad de selección de los usuarios mediante la generalización en las aplicaciones de modelos de acceso a contenidos y servicios que suponen un coste diferido, limitado o complementario. Son modelos basados en publicidad, en versiones lite o freemium, que hacen posible probar una aplicación (total o parcialmente) antes de afrontar su coste. Estos modelos no sólo subsidian el aprendizaje de la interfaz por el usuario (Fransman, 2014), sino que también le permiten resolver eficazmente la incertidumbre coste/beneficio, un elemento clave en las industrias del contenido y de servicios innova- 
dores. Con ello generan un proceso de selección por el que sólo se instala en los dispositivos una parte de la enorme población de aplicaciones. Esa población seleccionada se convierte, sin embargo, en la principal interfaz de acceso a contenidos y servicios en movilidad (Khalaf, 2014). Por esa misma lógica, las aplicaciones dejan de ser un producto acabado y cerrado y devienen un servicio dinámico que, en sucesivas versiones y actualizaciones, transforma su catálogo de funciones y su condición de interfaz.

Más allá del debate sobre la supuesta muerte de la Web, la idea motriz que subyacía en el artículo de Anderson y Wolff es que un cambio en la interfaz dominante supone un cambio en la lógica misma de internet. En el caso concreto del contenido digital, este cambio supone el paso de una lógica del ver -que prima el consumo de contenido como acceso-, a una lógica del hacer -que entiende el contenido como una materia prima para la expresión personal y para la interacción social-.

\section{Marco teórico y metodología}

\subsection{Las aplicaciones móviles como interfaz de con- tenido}

Las aplicaciones móviles o apps son piezas de software diseñadas para ser instaladas y utilizadas en dispositivos móviles, que se adaptan a las limitaciones de estos dispositivos pero también permiten aprovechar sus posibilidades tecnológicas (por ejemplo, la localización para servicios adaptados al contexto o el acelerómetro en algunos videojuegos) (Allen, 2003).

Se caracterizan por su economía funcional y por la importancia de su diseño de interfaz (Humphreys; Von-Pape; Karnowski, 2013). Están diseñadas para realizar funciones concretas, como presentar la información del tiempo en ciudades seleccionadas o comparar los precios de un producto en comercios cercanos. En ellas el diseño de interfaz debe ser intuitivo y natural, para responder adecuadamente a los requisitos de rapidez, ubicuidad y conveniencia propios de la movilidad (Joyce; Lilley, 2014).

Por contenido nos referimos a todo tipo de texto (escrito, icónico, audiovisual, hipermedia...). Tradicionalmente el término hace referencia al producto característico de las industrias culturales, sea en el ámbito del entretenimiento o en el de la información. Sin embargo, con la web 2.0 y el entorno móvil enfocado a las comunicaciones interpersonales, las interacciones entre usuarios en entornos sociales deben ser también entendidas como formas dialógicas de contenido (Jenkins; Ford; Green, 2013).

Las acciones sobre contenido digital pueden agruparse en cuatro grandes categorías:

- creación/edición: producir unidades de texto y modificar su estructura o aspecto;

- gestión: almacenar, organizar, agrupar e interrelacionar unidades textuales;

- reproducción: presentar o hacer accesible una unidad textual para su lectura;

- comunicación: incluir unidades textuales en cadenas dialógicas que pueden constituir ellas mismas textos dotados de sentido propio.
El software convencional ha tendido a tratar estas acciones como compartimentos separados. Encontrábamos así potentes herramientas centradas en la edición de textos o imágenes, la creación musical o la edición de vídeo, además de reproductores de diversa índole diseñados para visualizar o presentar todo tipo de contenidos. También herramientas de comunicación que permitían formas de interacción más o menos sincrónicas entre usuarios en las que el contenido jugaba un papel residual (Manovich, 2013). Con la transición de un internet centrado en los contenidos (web 1.0) a un internet de relaciones (web 2.0), empezó a darse la coordinación de esas funciones, especialmente en la comunicación: los contenidos dejaban de ser algo colateral -adjuntos a la conversación- para formar parte de ella (Jenkins; Ford; Green, 2013). Actualmente la integración entre contenido, computación y comunicación constituye de hecho una seña de identidad característica de los nuevos entornos ubicuos (Aguado; Martínez, 2014).

La taxonomía propuesta identifica las tendencias evolutivas en el desarrollo de aplicaciones de contenido

\subsection{Argumento y objetivos: cuando la interfaz rede- fine el contenido}

La hipótesis de partida de este trabajo busca situar en el contexto específico de los contenidos móviles el argumento de Anderson y Wolff (2010):

- el cambio en la interfaz de uso/consumo del contenido favorece un cambio en los modos de consumo del contenido y en la concepción última del mismo;

- ese cambio apunta hacia una mayor capacidad de intervención sobre el contenido por el usuario y de una mayor integración del contenido en las interacciones sociales.

A partir de aquí y desde un planteamiento descriptivo, se derivan dos objetivos principales:

- fundamentar una tipología funcional de las aplicaciones móviles de contenido y un modelo consecuente de análisis de su evolución funcional;

- comprobar en qué medida la evolución funcional de las aplicaciones móviles de contenido camina hacia una aceleración de la fusión entre herramienta y contenido, y entre los distintos ámbitos funcionales del contenido.

\subsection{Hacia una tipología de apps de contenido}

Comprender la diversidad cambiante de las aplicaciones móviles exige un trabajo previo de clasificación que permita discernir tipos y familias. Tanto más en el caso de las aplicaciones móviles de contenido, donde la mezcla entre tipos de contenido, funciones y servicios supone un especial desafío.

Buena parte de las clasificaciones existentes de apps de contenido responde a criterios de gestión comercial de categorías (utilizados en las tiendas de aplicaciones) o bien a criterios de género/propósito (publicidad, información, entretenimiento...). Aunque estos criterios pueden contribuir a facilitar su acceso o visibilidad, carecen de congruencia 


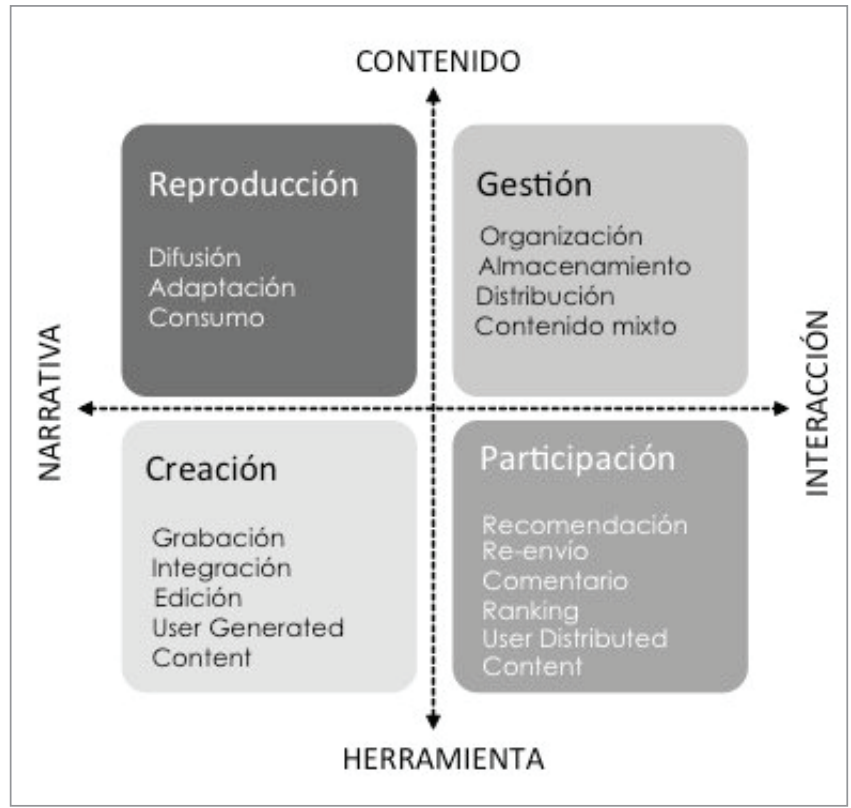

Figura 1. Clasificación funcional de aplicaciones móviles de contenido. Adaptación a partir de Aguado y Martínez, (2014) y Scolari, Aguado y Feijóo (2013).

suficiente para articular su análisis (Scolari; Aguado; Feijóo, 2012).

En trabajos anteriores (Aguado; Martínez, 2014; Scolari; Aguado; Feijóo, 2013) se ha desarrollado un modelo posicional de clasificación de aplicaciones móviles de contenido que ofrece dos ventajas analíticas:

- hace operativas las categorías de acciones sobre el contenido digital señaladas más arriba (creación, gestión, reproducción y comunicación);

- articula un espacio evolutivo para los tipos de aplicaciones, antes que categorías rígidas.

Esto permite analizar las polivalencias funcionales y otras formas de ambigüedad características sin generar incongruencias en la taxonomía.

El modelo propuesto (figura 1) se articula sobre dos dimensiones:

- vertical: oposición entre contenido (ver, acceder) y herramientas (hacer, transformar), con arquetipos instrumentales como la televisión y la videocámara, respectivamente;

- horizontal: oposición entre narrativas (articulación de relatos) e interacción (conversaciones), como extremos opuestos de la actividad discursiva del contenido, uno centrado en la narración, el otro en el diálogo.

Los ejes ortogonales definen un espacio conceptual donde las funciones predominantes (y por tanto, la interfaz) de las apps permiten diferenciar cuatro grandes áreas:

\section{Área de reproducción}

Aplicaciones cuyas funciones se centran en la reproducción de contenidos en los que la construcción narrativa tiene una dimensión relevante. En ella la capacidad de intervención sobre el contenido o la dimensión dialógica tienen un carác- ter secundario o no integrado. Las apps que adaptan contenidos propios de otros medios (televisión, música, libro, etc.) al entorno móvil tienden a ubicarse en este espacio. Son ejemplos Clan TVE, BBC iPlayer o The New York times.

\section{Área de creación}

Aplicaciones centradas en la construcción de distintas formas de textos narrativos (escrito, icónico, vídeo, sonido...). Son el trasunto móvil de los clásicos editores (de texto, audio, vídeo...) que permiten crear e integrar contenidos. A diferencia de sus homólogos de sobremesa, tienen un carácter simplificado, predefinido y modular. Por ejemplo, los editores fotográficos han sustituido la complejidad de las herramientas integradas (gestión del color, enfoque, luminosidad, etc.) por filtros con acabados predefinidos. Esto favorece la extensión del modelo de negocio (la venta de distintos tipos de filtro, por ejemplo) y la integración de otras funciones (fundamentalmente de interacción social).

\section{Área de gestión}

Aplicaciones móviles dirigidas a la organización, almacenamiento y distribución de contenido de diversa índole (propio de los medios y/o creado por los usuarios). En el entorno de sobremesa buena parte de estas funciones forma parte de la interfaz del sistema operativo (gestión de archivos). En el entorno móvil es necesario diseñar herramientas específicas separadas. Abarca aspectos como gestión de las bibliotecas de medios, creación de listas de reproducción, agregación y curación de contenidos, etc. Las funciones relacionadas con la gestión de contenido se integran de forma natural con el comercio online y los canales de distribución digitales del contenido, de modo que algunas aplicaciones señaladas constituyen a la vez sitios de comercio electrónico y herramientas de gestión de contenidos. Los propios canales de distribución de las grandes plataformas (iTunes, App Store o Google Play) adoptan este modelo, incorporando la inteligencia de datos de las compras del usuario como elementos de valor añadido en la gestión del contenido.

\section{Área de participación}

Aplicaciones móviles orientadas a la interacción comunicacional entre usuarios (mensajería y redes sociales). Estos espacios de diálogo integran de modo creciente el contenido en prácticas sociales a través de las cuales los usuarios presentan sus identidades (Papacharissi, 2012), convirtiendo de hecho a los usuarios en distribuidores eficaces del contenido (Noguera-Vivo et al., 2013).

\section{Los resultados muestran una evolución hacia la integración multifuncional en las diferentes aplicaciones móviles}

\subsection{Propuesta de análisis funcional de las aplicacio- nes de contenido móvil}

El desarrollo del modelo conceptual propuesto identifica la orientación funcional de las apps de contenido móvil respecto de los ámbitos de acción posibles sobre el contenido (el contenido, el relato, la herramienta, la conexión/interacción con otros). La estructura de los datos referidos en 
Tabla 1. Items de la versión 2.0 de Flipboard.

\begin{tabular}{|l|}
\hline $\begin{array}{l}\text { Aplicación: FlipBoard } \\
\text { Versión 2.0. 27/03/2013. Desarrollador: FlipBoard }\end{array}$ \\
\hline $\begin{array}{l}\text { 1. Ahora puedes recopilar y guardar contenidos en tus propias revis- } \\
\text { tas pulsando el botón + }\end{array}$ \\
\hline 2. Tus revistas son públicas pero se pueden hacer privadas \\
\hline $\begin{array}{l}\text { 3. Utiliza el nuevo marcador para añadir contenidos a tu revista desde } \\
\text { el navegador }\end{array}$ \\
\hline $\begin{array}{l}\text { 4. Recibe notificaciones de FlipBoard cuando a la gente le gusta, } \\
\text { comenta o se suscriba }\end{array}$ \\
\hline 5. Envía por correo o comparte tus revistas en Facebook, Twitter, G+... \\
\hline 6. Rendimiento y carga de página mejorado \\
\hline
\end{tabular}

Fuente: AppStore, 2015

el App Store de Apple/iOS permite acceder a un corpus de descripciones precisas sobre la evolución funcional de estas aplicaciones: el historial de actualizaciones recoge las distintas versiones de una app a lo largo del tiempo y las innovaciones o aportes funcionales que cada una pone en juego, remontándose a versiones entre uno y dos años anteriores a la última accesible. Cada versión incluye una lista detallada de los cambios que incorpora. Antes que un conjunto de datos neutros, se trata de una descripción que los desarrolladores hacen a los usuarios de las mejoras de su producto, y por tanto, elaborada en los términos de una experiencia de uso reconocible.

La tabla 1 presenta a modo de ejemplo los items de la versión 2.0 de Flipboard -un agregador de contenidos con rasgos de red social que cuenta con 50 millones de usuarios registrados-.

Sobre esta base se llevó a cabo una adaptación del análisis de contenido tomando los items de las actualizaciones como unidades de análisis (por sus características de concisión y secuencialidad) y la estructura del modelo de clasificación como eje para las categorías. Los items no específicamente relacionados con el contenido han sido descartados (por ejemplo, enunciados genéricos del tipo: corrección de errores, o mejora de rendimiento). Los relacionados con el contenido se han codificado conforme a tres niveles (nulo, intermedio y absoluto, entre 0 y 1 ) que puntúan su relación significativa con cada uno de los siguientes aspectos:

- reproducción o visualización de contenido (contenido);

- realización de acciones sobre contenido, como editar o modificar (herramienta);

- organización de contenido (gestión);

- comunicación con otros usuarios (interacción).

El análisis permite así describir la evolución de las funciones de una aplicación -a través de las sucesivas versiones- en el marco de las cuatro categorías consideradas (reproducción, creación, gestión, participación).

Los datos obtenidos pueden expresarse en un diagrama radial que permite visualizar de forma concisa la evolución de las dimensiones funcionales consideradas; esto es, si una aplicación evoluciona hacia la multidimensionalidad funcional y en qué medida lo hace en cada uno de los ámbitos funcionales considerados (figuras 3 a 7).
Tabla 2. Distribución por categorías de la selección muestral de aplicaciones de contenido móvil

\begin{tabular}{|l|l|l|l|}
\hline \multicolumn{1}{|c|}{ Reproducción } & \multicolumn{1}{c|}{ Creación } & \multicolumn{1}{c|}{ Gestión } & Participación \\
\hline The New York times & PicsArt & FlipBoard & Twitter \\
\hline Clash of clans & Garage band & Runtastic & Google+ \\
\hline YouTube & Clipper & Sing karaoke & Pinterest \\
\hline
\end{tabular}

Entre noviembre y diciembre de 2014 se realizó un primer test de validación sobre 4 apps cualitativamente significativas de los tipos propuestos (NYT, categoría de reproducción; Garage band, herramienta; FlipBoard, gestión; Twitter, participación) para las versiones acumuladas del último año. Los resultados fueron consistentes para codificadores diferentes.

Posteriormente se ha aplicado el modelo de análisis a una muestra de 12 aplicaciones (tabla 2), seleccionada conforme a criterios de relevancia cualitativa, discriminando para cada caso entre las cinco aplicaciones (gratuitas o de pago) más descargadas de su categoría (según las propias métricas de iOS App Store). Se han excluido apps populares debido a la ausencia de información específica sobre la innovación funcional de las actualizaciones (caso de Facebook). Siempre que ha sido posible, se ha optado por seleccionar productos de distinto tipo dentro de una misma categoría:

- medios o juegos en reproducción;

- herramientas de edición de distintos tipos de texto en creación;

- gestores de contenido, agregadores/recomendadores, y gestores de datos obtenidos del contexto en la categoría de gestión;

- redes sociales para participación.

Las áreas de gestión y participación concentran el grueso de la evolución funcional de agregadores como Flipboard

Las aplicaciones se han asignado a cada categoría a partir de la ponderación de los atributos funcionales que proporcionan los desarrolladores en la App Store. Algunas presentan ya en su planteamiento original un alto grado de cohesión multifuncional: YouTube por ejemplo, es al mismo tiempo una herramienta de reproducción de vídeo, un buscador, una red social o una herramienta de edición y gestión de contenido. La propuesta de una clasificación a partir de un mapa de posiciones permite ubicar los objetos de análisis dentro de una categoría pero en áreas próximas o intersecadas con las de otras categorías, con lo que se da cuenta de manera más eficaz de la condición multidimensional de las funciones sobre el contenido en las aplicaciones móviles.

Para la muestra seleccionada se ha extraído el historial de actualizaciones disponible en la App Store, tomándose un período de dos años, desde marzo de 2013 a marzo de 2015. Esto ha generado un corpus de 646 items funcionales válidos distribuidos en 153 versiones, lo que supone cerca de 28 items funcionales nuevos o modificados en 7 versiones por aplicación y año. Este dato por sí solo da una idea del 


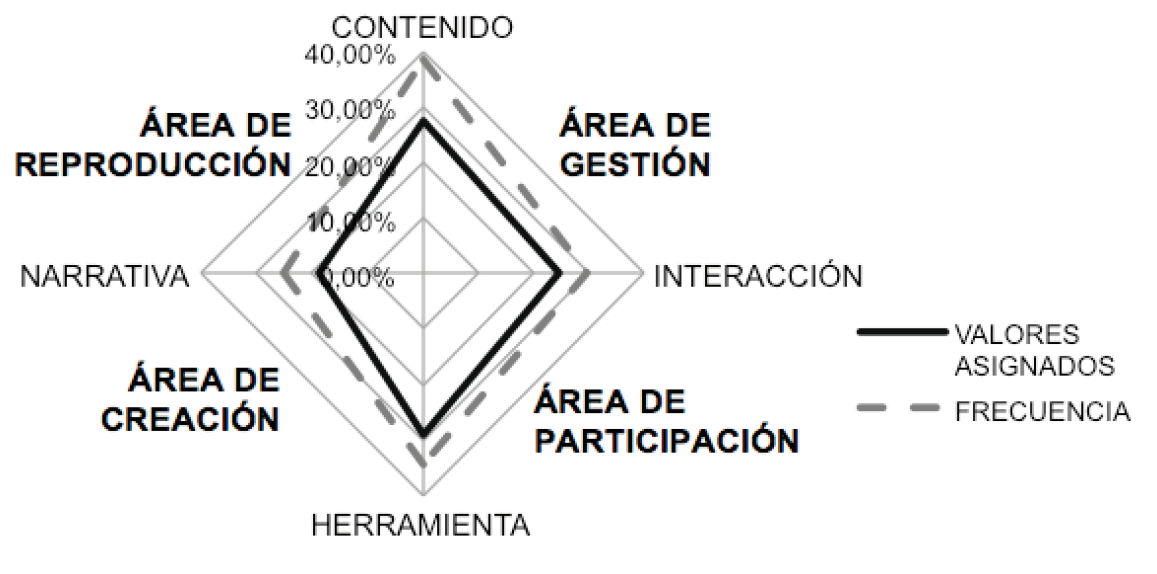

Figura 2. Gráfico de resultados globales para valores asignados y frecuencia (en porcentajes). tegoría puntúa en cada una de las actualizaciones analizadas, expresada en la línea discontinua de la figura 2).

Las zonas en que las líneas de ambas medidas se encuentran más próximas son los ámbitos en los que la evolución es más concentrada en el tiempo: a diferencia de otras categorías (como ocurre con contenido), en el caso de herramienta e interacción, una menor frecuencia o un número menor de veces que puntúan aparece asociado a un mayor valor absoluto (una puntuación mayor). Esto dinamismo de lo que Fransman (2014) ha señalado como un sistema de innovación emergente. Aunque la muestra ofrece una representatividad limitada -que podrá ser desarrollada en posteriores investigaciones-, los resultados permiten validar el modelo y ofrecen matices interesantes sobre la evolución funcional de las aplicaciones de contenido móvil.

Las aplicaciones de medios centran su evolución en el acceso al contenido, desarrollando de forma muy limitada el ámbito de las interacciones sociales y gestión del contenido

\section{Resultados}

Los resultados obtenidos muestran una evolución general hacia la integración multifuncional en las aplicaciones móviles, con porcentajes próximos al 30\% de los valores asignados para los ámbitos funcionales relacionados con: permite anticipar una cierta aceleración (concentración en el tiempo) y relevancia (valor) en la inclusión de funciones relativas a aspectos sociales sobre el contenido.

Esta tendencia no se distribuye de forma homogénea por los distintos tipos de aplicación considerados. En las aplicaciones de la categoría funcional de reproducción existen claras diferencias en cuanto a la intensidad y dirección de la evolución. Las aplicaciones de medios (The New York times) se caracterizan por centrar su evolución funcional en el acceso al contenido (45,2\%), desarrollando de forma muy limitada cierta multifuncionalidad en el ámbito de las interacciones sociales y gestión del mismo (figura 3). En el plano cronológico es interesante observar cómo en las versiones lanzadas en 2013 (4.0 a 4.0.3) se produce un salto cualitativo en las funciones de interacción, que luego es compensado a lo largo de 2015 con el desarrollo de funciones de acceso y gestión del contenido.

El contraste con los videojuegos móviles como Clash of clans es manifiesto. Aquí los valores acumulados se concentran

- mejoras en el contenido (27,5\%);

- mejoras o modificaciones instrumentales (29,2\%);

- formas o procesos de interacción (24,6\%).

En términos globales las áreas funcionales más desarrolladas en las actualizaciones entre marzo de 2013 y marzo de 2015 son las de gestión y de participación (línea continua en el gráfico de la figura 2).

El modelo permite también apreciar el grado de distribución o de concentración de la evolución en los distintos ámbitos funcionales (reproducción, creación, gestión y/o participación) a lo largo del período estudiado. Éste se obtiene comparando los valores asignados (la puntuación total de los items de las actualizaciones en cada categoría, expresada en la línea continua de la figura 2) con la frecuencia (número de veces que una ca-

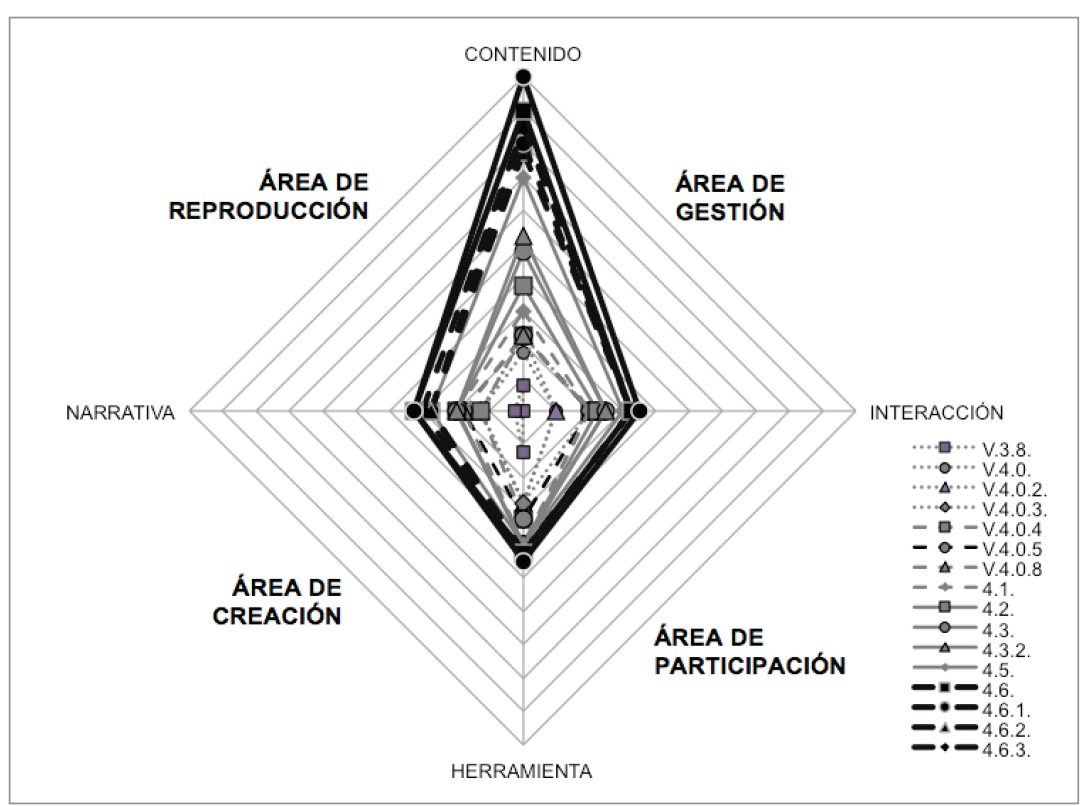

Figura 3. Gráfico de resultados de The New York times 


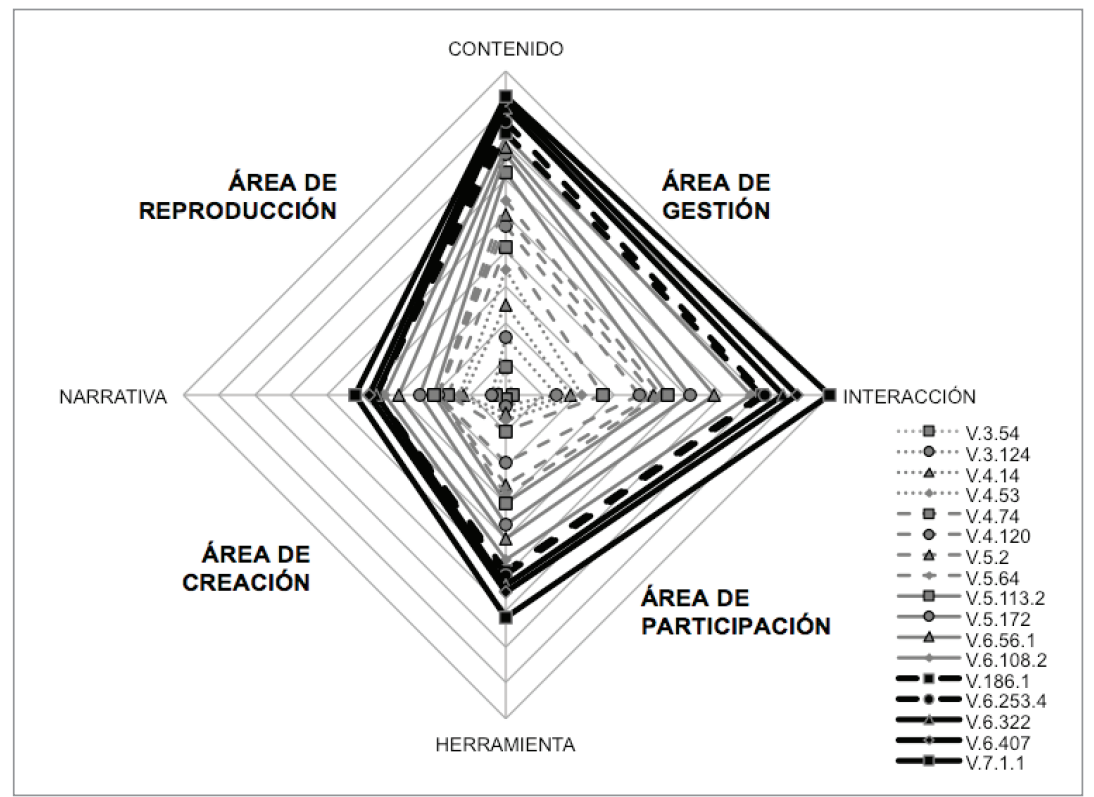

Figura 4. Gráfico de resultados de Clash of clans

en las categorías funcionales de contenido $(29,4 \%)$ e interacción $(30,1 \%)$ con una evolución marcadamente regular en las sucesivas versiones (figura 4). Claramente el relato y la capacidad de creación son ámbitos funcionales secundarios frente a la preponderancia del contenido visual (gráficos y estructura) y las funciones sociales del juego.

El caso de YouTube es también singular por su polivalencia funcional, como ya se ha comentado. La evolución funcional concentra las innovaciones en las categorías de contenido $(33,3 \%)$, narrativa $(30,6 \%)$ y herramienta $(22,2 \%)$ con sólo un $13,9 \%$ en interacción. La aplicación ha intensificado por tanto en los últimos dos años sus capacidades en la gestión, acceso y edición de contenido.

Las apps del área de gestión tienen un claro protagonismo en el conjunto de las tendencias evolutivas analizadas. El caso de FlipBoard resulta especialmente sintomático. Nacida como una aplicación de agregación de contenido con ciertas funciones sociales (integraba contenidos procedentes de las cuentas de Twitter y FaceBook del usuario), ha desarrollado posteriormente sus funciones hacia los ámbitos de herramienta con un $28,2 \%$ (creación de revistas personales, nuevas formas de integrar y gestionar la curación de contenido) e interacción con un 30,3\% (suscriptores, colaboradores, contactos, etc.). El contenido con un $21,8 \%$ es también otro foco de evolución. El resultado es que las áreas de gestión y participación concentran el grueso de su evolución funcional (figura 5). La correlación se reproduce en las otras dos apps analizadas para la categoría de gestión (Runtastic y Sing karaoke).

Las aplicaciones del área de creación son las que presentan un menor grado de evolución hacia la integración de ámbitos funcionales. Se trata de aplicaciones que, como en el caso de PicsArt (edición gráfica), Garage band (edición de audio) o Clipper (edición de vídeo), concentran su evolución predominantemente sobre el ámbito que las define (la intersección entre narrativa y herramientas). Este rasgo favorece su deriva hacia modelos más próximos al comercio electrónico en los que las funciones modularizadas (filtros de fotografía, clips de audio o efectos de vídeo) devienen objeto de actividad comercial. Circunstancialmente existe cierta tendencia a la inclusión de funciones sociales (relacionadas con la compartición de obras y con la valoración en rankings de los items comerciales como filtros o clips), más patente en el caso de la edición gráfica (un 11,5\% en PicsArt) que en el audio (un 3\% en Garage band). Obsérvese en el gráfico de PicsArt que la estructura resultante de su evolución es simétricamente inversa a la de The New York times (figura 6).

Junto a las apps de gestión, son las de participación las que mayor convergencia multifuncional concitan. No en vano las redes sociales suponen un $24 \%$ del uso de las aplicaciones móviles, sólo por detrás de los videojuegos (31\%) (Khalaf, 2014). Dos tendencias claras afloran en la evolución de las redes sociales móviles:

- diversificación y enriquecimiento de las funciones asociadas con la interacción (nuevas formas de interacción y de seguimiento);

- integración creciente del acceso al contenido.

Los valores asignados a las funciones de interacción (30,2\% en Twitter, 28,5\% en Google+, 29,2\% en Pinterest) y de contenido (27,1\% en Twitter, $29,9 \%$ en Google+, $41,7 \%$ en Pinterest) destacan con diferencia, arrastrando los asignados a

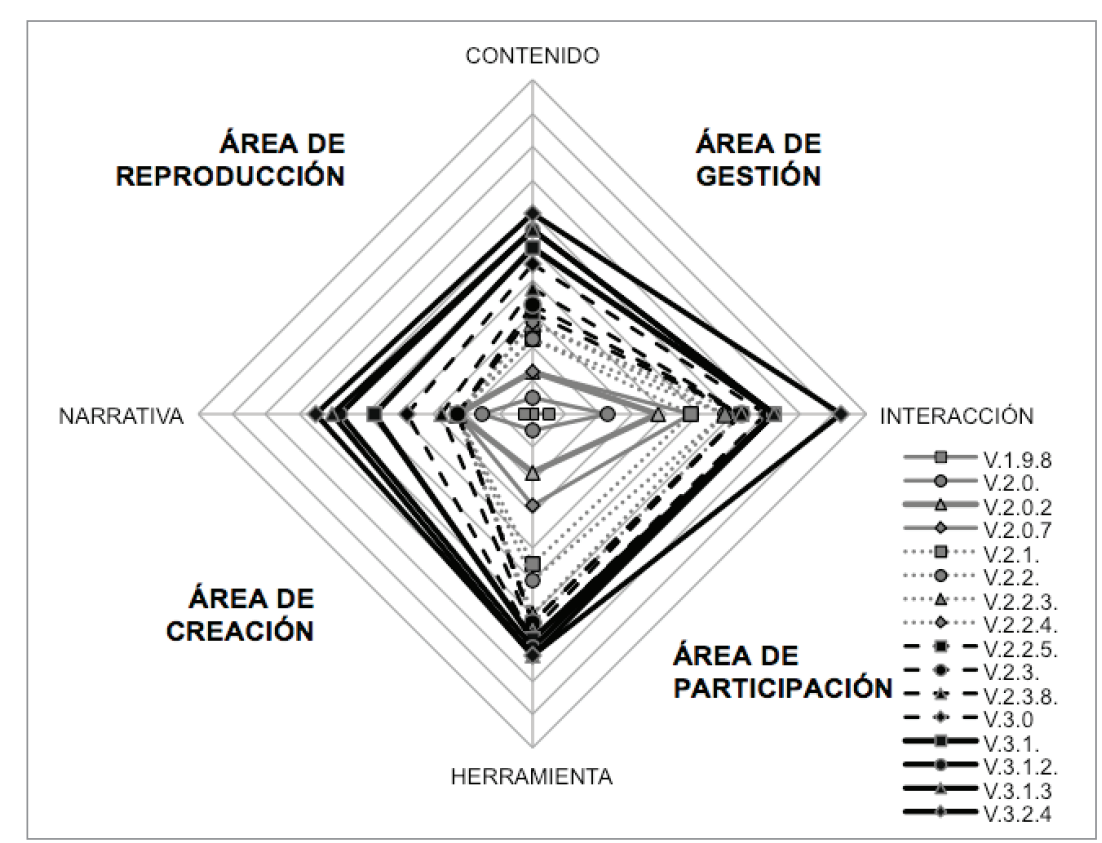

Figura 5. Gráfico de resultados de FlipBoard 


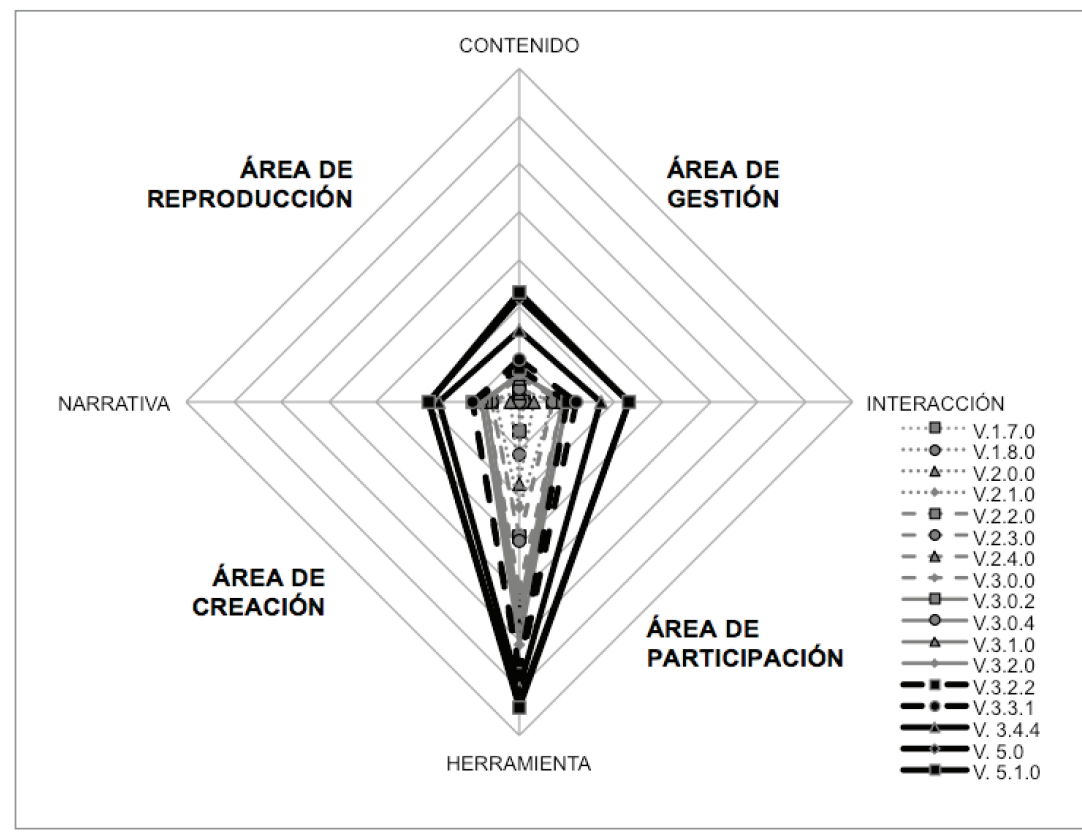

Figura 6. Gráfico de resultados de PicsArt

herramientas (capacidad de edición de contenido) $(29,2 \%$ en Twitter, $19,7 \%$ en Google+, $20,8 \%$ en Pinterest). El caso de Twitter resulta ilustrativo de estas tendencias, con las áreas de gestión (intervención sobre contenido) y participación (comunicación) como sus principales ámbitos funcionales de expansión. Obsérvese en este sentido la similitud entre la estructura de la evolución funcional de FlipBoard y Twitter, sólo diferenciada por el mayor peso del relato en la evolución de la primera.

\section{Discusión y conclusiones}

Ante la necesidad de comprender un fenómeno complejo y dinámico como las nuevas formas de interfaz de contenido en las plataformas móviles, se hace especialmente necesaria una taxonomía flexible pero coherente que permita dar cuenta de la naturaleza evolutiva de su propio objeto (Flora; Wang; Chande, 2014). La dimensión estratégica y ubicua del contenido en el nuevo entorno digital hace extensivo el argumento al ámbito de las aplicaciones móviles de contenido.

El modelo propuesto resulta útil para representar y comprender las intersecciones y mestizajes entre categorías y permite una aproximación al análisis funcional de las aplicaciones de contenido basada en el peso de los distintos ámbitos funcionales (representación, gestión, creación y comunicación) en el diseño de la interfaz. La taxonomía propuesta sustenta un modelo de análisis descriptivo que identifica las tendencias evolutivas en el desarrollo de aplicaciones de contenido, permitiendo elaborar un corpus de datos cuya correlación con otras herramientas metodológicas y objetos de análisis (experiencia de uso, usabilidad, comportamiento del consumidor, etc.) puede arrojar luz sobre un elemento clave del nuevo ecosistema tecnológico (De-Prato; Sanz; Simon, 2014).

El análisis realizado pone de relieve que las aplicaciones de gestión de contenido y las redes sociales son las que mayor integración funcional alcanzan. Primero, porque articulan su catálogo funcional en torno a la interacción y el contenido. Segundo, porque incorporan de manera subordinada funciones de edición y creación de contenido. Así, la integración de Google+ con las capacidades de edición de alguno de sus servicios web, o la asociación de Twitter con Vine o, más recientemente, con Periscope (ambos para la edición y transmisión de vídeo en formato ligero), ejemplifican una tendencia a la integración del contenido como materia prima de las conversaciones sociales. En esta misma tendencia cabe inscribir la evolución de los videojuegos móviles, claramente escorada hacia lo social (Feijóo et al., 2012), donde el contenido y la conversación se instrumentalizan recíprocamente.

En el extremo opuesto las apps vinculadas a medios convencionales y las de creación y edición de contenidos circunscriben su evolución funcional al ámbito que las define. En las aplicaciones de medios conviene tener en cuenta que parte de la multidimensionalidad funcional se encuentra externalizada en estrategias multicanal. Al tratarse de productos o servicios con presencia impresa o televisiva, web, en agregadores, etc., pueden desagregar las funciones en distintos canales y productos para optimizar la circulación de sus audiencias (Smith, 2015). No ocurre así con las aplicaciones y servicios de edición, cuya tendencia es la comoditización del saber hacer instrumental (fotográfico, musical, de vídeo...),

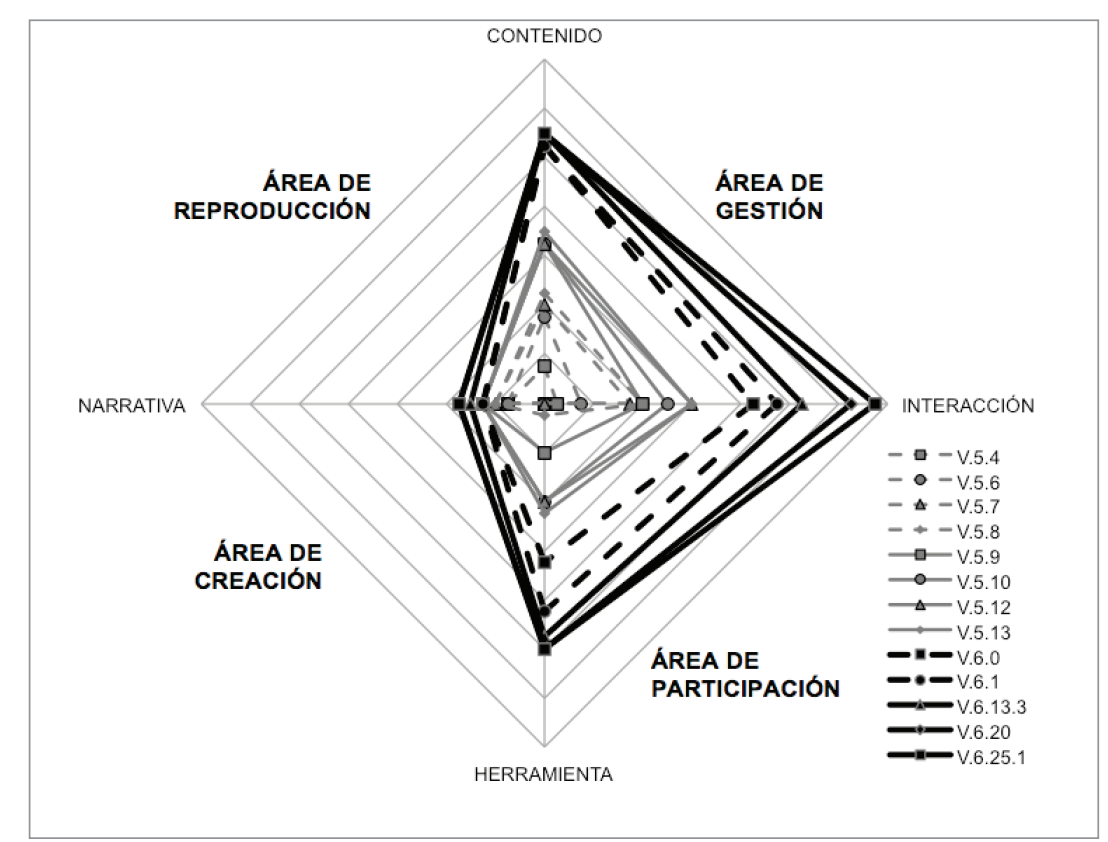

Figura 7. Gráfico de resultados de Twitter 
su transformación en una suerte de juego creativo y su comercialización como estructura de capas o items funcionales (filtros fotográficos, riffs de guitarra, efectos de vídeo, etc.). La estructura simétrica inversa de los resultados para ambos tipos de aplicaciones se corresponde con modelos de negocio similares (pago por contenido en NYT y pago por ítem funcional en PicsArt). Es posible deducir aquí cierto grado de correlación entre el modelo de negocio y la concentración funcional que puede ser objeto de investigaciones posteriores.

Las aplicaciones móviles de contenido han evolucionado en los dos últimos años hacia una creciente convergencia funcional en la que las funciones tipificadas en los ámbitos de gestión y participación adquieren un carácter determinante de la interfaz. Dicha evolución pone en juego una concepción activa y social del contenido, que deja de ser un objeto de consumo finalista para convertirse en un lenguaje, en un recurso. El contenido ya no es sólo algo que se lee, se ve o se escucha: es cada vez más algo sobre lo que hacer cosas (compartir, enlazar, comentar, puntuar, modificar...) y algo que se integra en las dinámicas de interacción social (se reenvía, se enlaza, se comenta, se parodia...).

\section{Bibliografía}

Aguado, Juan-Miguel; Martínez, Inmaculada J. (2014) "The relationship is the medium: Understanding media in a mobile age". En: Katz, James. Living inside mobile social information. Boston, MA: Boston University Press, pp. 77-108. ISBN: 9781570741289

http://sites.bu.edu/cmcs/files/2014/01/Living-InsideMobile-Social-Information.pdf

Allen, Jonathan P. (2003). "The evolution of new mobile applications: a sociotechnical perspective". International journal of electronic commerce, v. 8, n. 1, pp. 23-36.

Anderson, Chris; Wolff, Michael (2010). "The Web is dead, long life to the internet", Wired, 17 agosto.

http://www.wired.com/2010/08/ff_webrip

De-Prato, Giuditta; Sanz, Esteve; Simon, Jean-Paul (2014). Digital media worlds. The neew economy of media. New York: Palgrave-Macmillan. ISBN: 9781137344243

Feijóo, Claudio; Gómez-Barroso, José-Luis; Aguado, JuanMiguel; Ramos, Sergio (2012). "Mobile gaming: Industry challenges and policy implications". Telecommunications policy, v. 36, n. 3, pp. 212-221.

http://oa.upm.es/15635/2/INVE_MEM_2012_129874.pdf

http://dx.doi.org/10.1016/j.telpol.2011.12.004

Flora, Harleen K.; Wang, Xiaofeng; Chande, Swati V. (2014). "An investigation on the characteristics of mobile applications: A survey study". International journal of information technology and computer science (ljitcs), v. 6, n. 11, pp. 21-27. http://www. mecs-press.org/ijitcs/ijitcs-v6-n11/IJITCSV6-N11-3.pdf

http://dx.doi.org/10.5815/ijitcs.2014.11.03

Fransman, Martin (2014). Models of innovation in global ICT firms: The emerging global innovation ecosystems.
EC-JRC Science and policy reports. European Commission. ISBN: 9789279395338

https://ec.europa.eu/jrc/sites/default/files/jrc90726.pdf http://dx.doi.org/10.2791/10679

Humphreys, Lee; Von-Pape, Thilo; Karnowski, Veronika (2013). "Evolving mobile media: Uses and conceptualizations of the mobile internet". Journal of computer-mediated communication, v. 18, n. 4, pp. 491-507. http://dx.doi.org/10.1111/jcc4.12019

Jenkins, Henry; Ford, Sam; Green, Joshua (2013). Spreadable media: creating value and meaning in a networked culture. New York: NYU Press. ISBN: 9780814743508 http://dx.doi.org/10.1111/jpcu.12110

Joyce, Ger; Lilley, Mariana (2014). "Towards the development of usability heuristics for native smartphone mobile applications". In: Marcus, Aaron (ed.). Design, user experience, and usability. Theories, methods, and tools for designing the user experience. Lecture notes in computer science, v. 8517, pp. 465-474.

http://dx-doi.org/10.1007/978-3-319-07668-3_45

Khalaf, Simon (2014). "Apps solidify leadership six years into the mobile revolution". Flurry analytics, April 1.

http://flurrymobile.tumb/r.com/post/115191864580/appssolidify-leadership-six-years-into-the-mobile

Manovich, Lee (2013). Software takes command. New York: Bloomsbury. ISBN: 9781623567453

Meeker, Mari (2014). "2014 Internet trends". KPCB, May 28. http://www.kpcb.com/blog/2014-internet-trends

Noguera-Vivo, José-Manuel; Bourdaa, Melanie; Villi, Mikko; Nyiro, Nora; De-Blasio, Emiliana (2013). "The role of the media industry when participation is a product". In: Carpentier, Nico; Schroder, Kim; Hallett, Lawrie (eds.). Transformations. Late modernity's shifting audience positions. New York: Routledge, pp. 172-190. ISBN: 9780415827362

Papacharissi, Zizi (2012). "Without you, I'm nothing: Performances of the self on Twitter". International journal of communication, v. 6, n. 18, pp. 1989-2006.

http://ijoc.org/index.php/ijoc/article/view/1484

Scolari, Carlos; Aguado, Juan-Miguel; Feijóo, Claudio (2012). Mobile media: Towards a definition and taxonomy of contents and applications. International journal of interactive mobile technologies, v. 6, n. 2.

http://dx.doi.org/10.3991/ijim.v6i2.1880

Scolari, Carlos; Aguado, Juan-Miguel; Feijóo, Claudio (2013). "Una ecología del medio móvil: contenidos y aplicaciones". En: Aguado, Juan-Miguel; Feijóo, Claudio; Martínez, Inmaculada J. (2013). La comunicación móvil: hacia un nuevo ecosistema digital. Barcelona: Gedisa, pp. 127-154. ISBN: 9788497847827

Smith, Aaron (2015). "U.S. smartphone use in 2015". Pew Research Center. Internet, science \& tech, April 1. http://www.pewinternet.org/2015/04/01/us-smartphoneuse-in-2015 


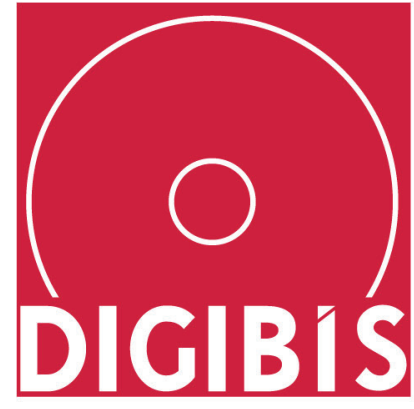

Digitalización enriquecida de fondo antiguo y patrimonial

Software de gestión para Bibliotecas, Archivos y Museos DIGIBIB ${ }^{\circ}$ DIGIARCH $^{\circ}$ DIGIMUS ${ }^{\circ}$

\section{Recolector OAI-PMH DIGIHUB' de metadatos de diversos proveedores}

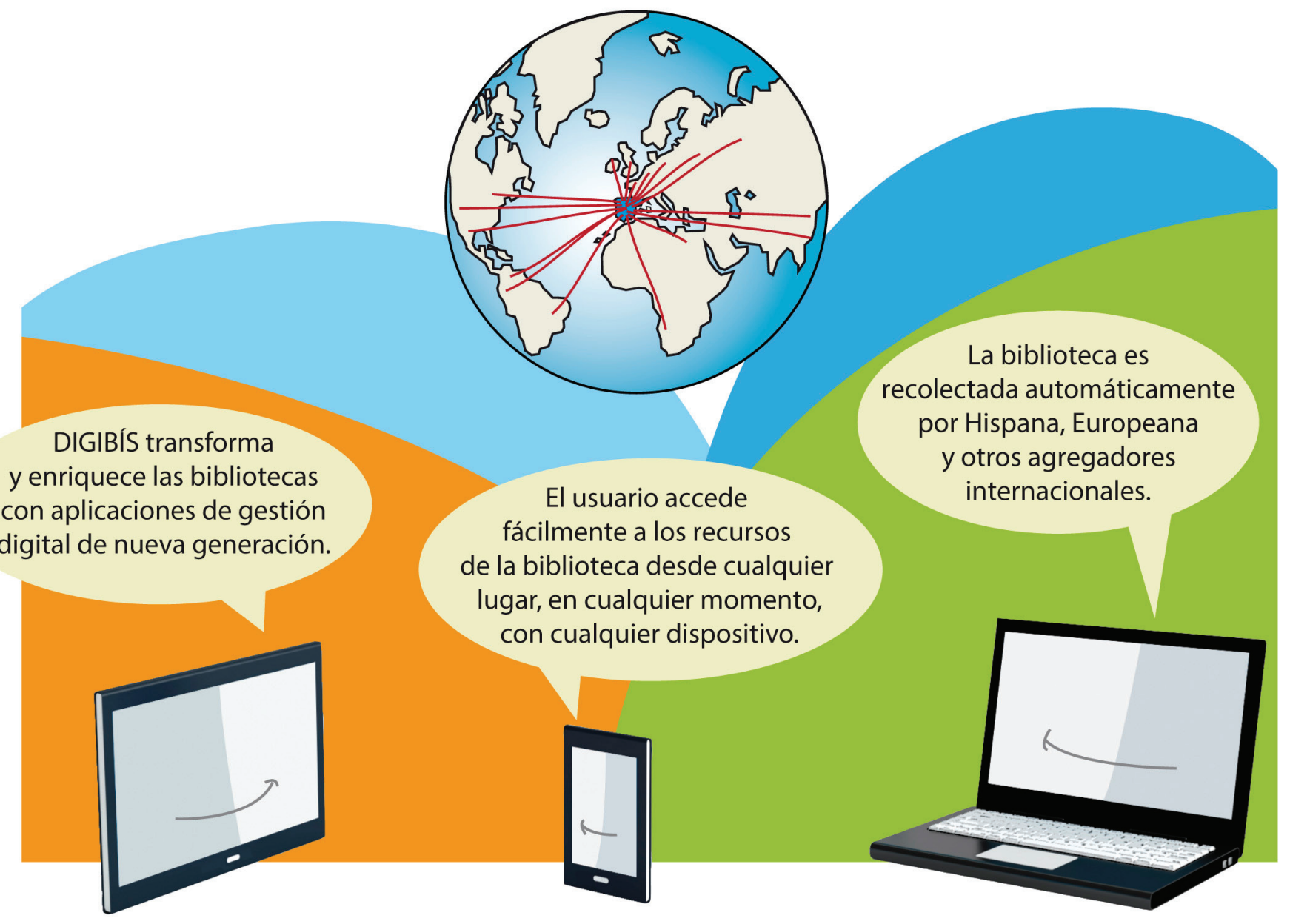

\section{¡Con estándares internacionales para un mundo enlazado y abierto!}

